\title{
DIDACTIC- Gamificación e inteligencia artificial como apoyo a los programas educativos virtuales
}

\author{
Martha Liliana Torres-Barreto ${ }^{a}$, Julieth Katherin Acosta-Medina ${ }^{\text {, }}$, Maira Camila \\ Paba-Medina $^{\mathrm{c}}$ \\ ${ }^{a}$ Universidad Industrial de Santander, Bucaramanga, Colombia, mltorres@uis.edu.co, ${ }^{b}$ Universidad \\ Industrial de Santander, Bucaramanga, Colombia, katheacosta19@gmail.com, ${ }^{\mathrm{c}}$ Universidad Industrial \\ de Santander, Bucaramanga, Colombia,mairacami@hotmail.com.
}

\begin{abstract}
Resumen
El abandono de los programas académicos es uno de los principales problemas para la educación superior, principalmente en la modalidad virtual. Por ejemplo, para Colombia la tasa de abandono en programas educativos virtuales es del $60 \%$. Esto se debe, principalmente, a características propias de esta modalidad como la soledad, falta de dinamismo en los cursos y deficiente conexión docente - estudiante. Para contrarrestarlo, se hace necesario aplicar estrategias innovadoras que aumenten la motivación de los estudiantes y mejoren los procesos educativos. En este contexto surge el proyecto DIDACTIC que consta de una herramienta didáctica motivacional basada en gamificación y una plataforma de contactabilidad, las cuales se incorporan en los sistemas de gestión de aprendizaje de los programas virtuales. En este artículo se presentan las fases de diseño, desarrollo y validación de DIDACTIC. Por una parte, la herramienta didáctica, la cual se materializa a través de plataforma de desarrollo Unity, busca aprovechar los beneficios de la gamificación para incrementar el engagement y la motivación de los estudiantes, apoyando los procesos de enseñanza y aprendizaje de habilidades transversales como las competencias ciudadanas. Por otro lado, la plataforma de contactabilidad busca predecir la deserción estudiantil a través de modelos machine learning y mediante configuración de inteligencia artificial establecer contacto con los estudiantes por medio de correo electrónico, llamadas o diferentes redes sociales como WhatsApp o Facebook, en momentos precisos, para brindar diferentes actividades de refuerzo o acompañamiento y así evitar su posible deserción. Con este proyecto se pretende utilizar las nuevas tecnologías para crear experiencias de aprendizaje personalizadas y eficientes.
\end{abstract}

Palabras clave: Gamificación, inteligencia artificial, educación virtual, motivación. 


\section{Introducción}

La educación virtual enfrenta grandes problemas relacionados con las altas tasas de abandono y las bajas tasas de finalización, estos inconvenientes debido, en parte, al modelo de enseñanza usado en esta modalidad educativa, el cual se encuentra centrado en el contenido y no en los usuarios (Sastre, Ortega-Arranz, Gómez-Sánchez, \& VillagráSobrino, 2019). Incluso, los estudiantes que no abandonan el sistema educativo y logran terminar sus cursos manifiestan altas tasas de aburrimiento y falta de interés, ya que argumentan que el proceso de aprendizaje es tedioso y poco efectivo (Alivernini \& Lucidi, 2011; Heublein, 2014; Lassibille \& Navarro Gómez, 2008). Para el caso de Colombia, el promedio de abandono en programas de educación superior presencial es del $40 \%$, mientras que la tasa de abandono en programas virtuales alcanza el 60\% (Ministerio de Educación Nacional de Colombia, 2017).

En diferentes investigaciones se ha encontrado que al implementar tendencias educativas en entornos virtuales de aprendizaje se obtienen mejoras significativas en indicadores como el nivel de abandono, la percepción de los estudiantes y las notas obtenidas por los mismos (Gonzalez, Gomez, \& Echeverri, 2017). De este modo, la aplicación de estrategias innovadoras activas se presenta como una alternativa para contribuir a solucionar parte de estas problematicas (Sastre et al., 2019).

En este contexto surge el proyecto DIDACTIC que consta de dos componentes principales: una herramienta llamada "Didactic City" la cual busca utilizar los principios de la gamificación para incrementar la motivación de los estudiantes, apoyando los procesos de enseñanza y aprendizaje de las competencias ciudadanas; y una plataforma de contactabilidad que ayuda a predecir la deserción estudiantil a través de modelos de machine learning, esta plataforma además, hace uso de la inteligencia artificial para establecer contacto con los estudiantes a traves del correo electrónico, llamadas o redes sociales, brindando diversas actividades de refuerzo y/o acompañamiento en los momentos clave de su proceso de aprendizaje con el fin de evitar su posible abandono del curso. Estos componentes se incorporan en los sistemas de gestión de aprendizaje (LMS) de los programas educativos virtuales. En el presente trabajo se presentan las fases de diseño, desarrollo y validación de DIDACTIC. 


\section{Metodología}

Por una parte, la herramienta didáctica gamificada "Didactic City" tiene un diseño centrado en el usuario, para este proceso se siguió la metodología propuesta por Kumar \& Herger, (2013), la cual consta de cinco pasos: (1) Perfiles de usuario, (2) Motivaciones de los usuarios, (3) Objetivos de aprendizaje, (4) Elementos de la gamificación, (5) Administración y monitoreo. Posterior a esto, su desarrollo se materializó utilizando lenguaje de programación C\# en el motor Unity 3D. Adicionalmente, para obtener mejores resultados, la herramienta se validó con grupos de usuarios mediante pruebas de usabilidad (Ver Figura 1).

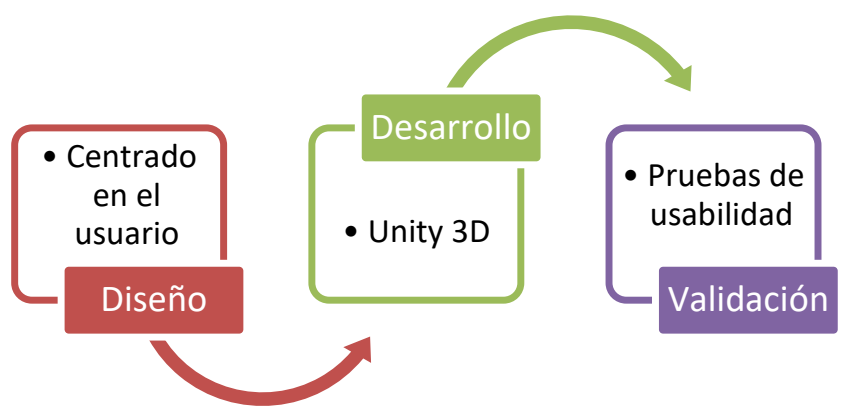

Fig. 1 Herramienta gamificada "Didactic City"

En cuanto a la plataforma de contactabilidad, en primer lugar se identificaron los modelos machine learning a construir. Para esto se realizó una revisión de literatura y un análisis de viabilidad. Los modelos seleccionados se desarrollaron utilizando el lenguaje de programación Python y se validaron mediante diferentes pruebas matemáticas. A partir de las predicciones de estos modelos y mediante una inteligencia artificial se establece comunicación con los estudiantes, en momentos determinados, con el objetivo de brindar actividades de refuerzo o acompañamiento (Ver Figura 2).

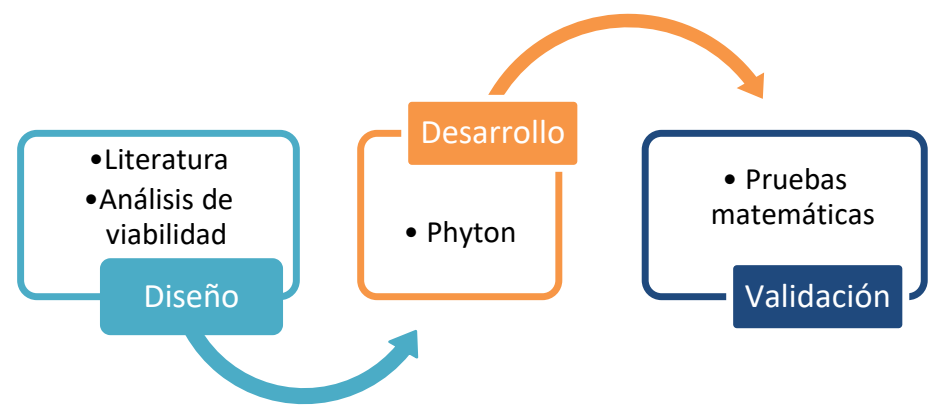

Fig. 2 Plataforma de contactabilidad 


\section{Resultados}

\subsection{Herramienta "Didactic City"}

A partir de una serie de entrevistas abiertas se identificaron cuatro perfiles de estudiantes de educación superior virtual: (1) Estudiante fantasma: solo ingresa a robarse los contenidos de los cursos, (2) Estudiante recién graduado: joven recién graduado de la secundaria que ve en la educación superior virtual una oportunidad para salir adelante, (3) Estudiante trabajador: trabaja para poder estudiar y (4) Estudiante de retos personales: individuos mayores de 50 años que en su tiempo libre buscan la realización personal. Para el contexto particular de este estudio predominan los estudiantes con perfil recién egresados. Para identificar sus principales motivaciones y necesidades, se realizaron mapas de empatía y journey maps, encontrando que estos individuos se motivan por canales de comunicación efectivos y por el acompañamiento constante por parte del docente.

Adicionalmente, esta herramienta busca apoyar los procesos de enseñanza y aprendizaje de las competencias ciudadanas, ya que estas tienen un papel fundamental en la sociedad pero siguen siendo un tema que necesita mejorar en la mayoría de los países (Acosta-Medina, Torres-Barreto, Álvarez-Melgarejo \& Paba-Medina, 2019), además estas son habilidades transversales a cualquier curso o programa educativo. Sin embargo, estas competencias según la educación cívica colombiana se clasifican en 7 categorías, por lo que se hizo necesario realizar una priorización de las mismas. Para esto se aplicó el método de ponderación de factores y el método Delphi, concluyendo que las habilidades comunicativas escritas y las cognitivas de conocimientos cívicos son las más importantes debido a su relevancia para la vida laboral y la ciudadanía en general (Ver Tabla 1).

Tabla 1. Priorización de competencias ciudadanas

\begin{tabular}{|c|l|c|}
\hline Cuartil & \multicolumn{1}{|c|}{ Competencias ciudadanas } & Puntaje \\
\hline 1 & Comunicativas escritas & 4,36 \\
& Cognitivas - conocimiento cívico & 4,21 \\
\hline 2 & Comunicativas orales & 3,65 \\
\hline 3 & Coognitivas - pensamiento sistémico & 3,11 \\
& Cognitivas - multiperspectivismo & 3,11 \\
& Cognitivas - argumentación & 2,92 \\
\hline \multirow{2}{*}{4} & Emocionales - manejo de emociones & 2,69 \\
& Emocionales - empatía & 1,94 \\
\hline
\end{tabular}

Con base en esta información, se seleccionaron los elementos de la gamificación a incluir en la herramienta, los cuales se basaron en el modelo conceptual propuesto por Werbach \& Hunter, (2012): (1) Dinámica: emociones, progresión y retroalimentación; (2) Mecánica: construcción; (3) Componentes: avatar, puntos, monedas, niveles, misiones, tablas de 
clasificación y barras de progreso. "Didactic City" se materializó usando el lenguaje de programación C\# en el motor de Unity 3D, esta herramienta gamificada consta de 8 niveles en los que los usuarios pueden construir una ciudad mientras resulven misiones de competencias ciudadanas (preguntas, dilemas morales y situaciones problema) (Ver Figura 3). Por cada misión cumplida el jugador recibe monedas customizables dentro de la ciudad y puntos de juego correspondientes a la gamificación activa, los cuales se suman con puntos de trabajo obtenidos por realizar actividades netamente académicas dentro del LMS, tales como presentar una tarea o participar en un foro (gamificación pasiva). La totalidad de los puntos determinan la posición de cada estudiante en la tabla de clasificación.
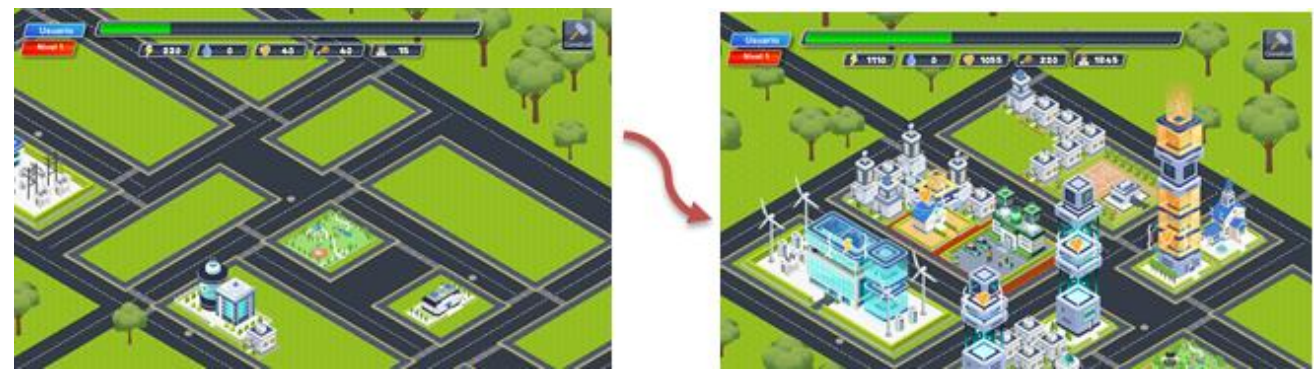

Fig. 3 Interfaz "Didactic City"

Para obtener mejores resultados se validó la herramienta gamificada con 72 estudiantes de educación virtual mediante pruebas de usabilidad. Cada prueba constaba de 30 minutos de juego en los que el usuario debía cumplir una serie de misiones en "Didactic City". Luego, cada participante se exponía a un cuestionario acerca de sus percepciones del juego, este instrumento evaluaba aspectos como la usabilidad (System Usability Scale), la experiencia de usuario (AttrakDiff), la estética de la experiencia (VisAWI) y la preferencia por el uso. Tal como se observa en la Figura 4, "Didactic City" presenta una usabilidad integrada aceptable, entre 71 y 100 puntos.

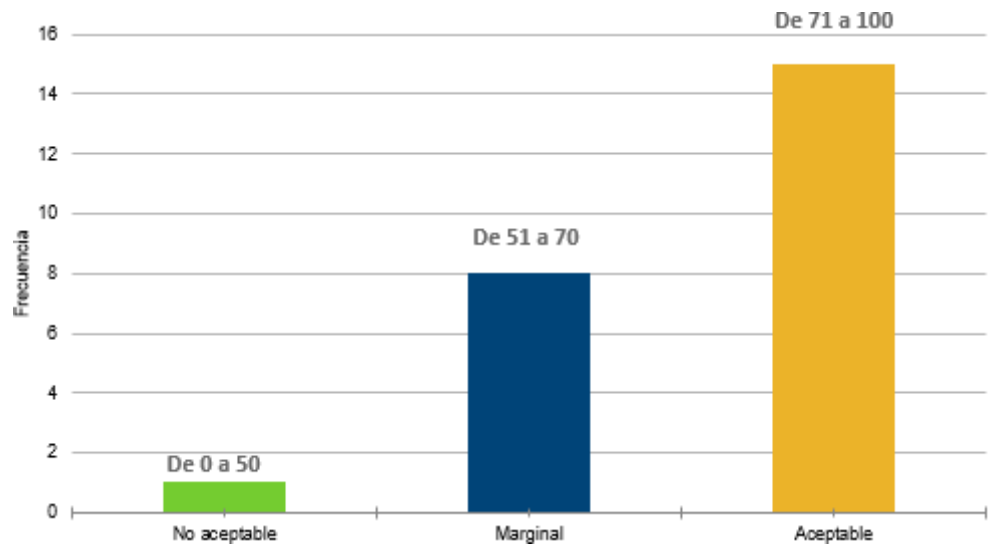

Fig. 4 Usabilidad integrada "Didactic City" 


\subsection{Plataforma de contactabilidad}

Según la literatura consultada, los modelos machine learning más usados para predecir el abandono estudiantil son los arboles de decisión, las redes neuronales, la regresión logística y el análisis discriminante. Sobre estos modelos se ejecutó un análisis de viabilidad teniendo en cuenta diversos factores, con el fin de establecer los más adecuados a usar (Ver Tabla 2). Totalizando los resultados se encuentra que en conjunto las redes neuronales y los arboles de decisión presentan el mejor valor de atributos, por lo que se construye un modelo que incluye ambos algoritmos. Para dicha construcción se utilizó el lenguaje de programación Phyton y algunas de sus librerías tales como: sklearn, pandas, numpy y scipy.

Tabla 2. Priorización de competencias ciudadanas

\begin{tabular}{|l|c|c|c|c|}
\hline \multicolumn{1}{|c|}{ Factores } & $\begin{array}{c}\text { Arboles de } \\
\text { decisión }\end{array}$ & $\begin{array}{c}\text { Redes } \\
\text { neuronales }\end{array}$ & $\begin{array}{c}\text { Regresión } \\
\text { logística }\end{array}$ & $\begin{array}{c}\text { Análisis } \\
\text { discriminante }\end{array}$ \\
\hline Usabilidad & 10 & 10 & 8 & 7 \\
\hline Requerimiento de datos & 9 & 9 & 7 & 7 \\
\hline Adaptabilidad & 9 & 9 & 9 & 9 \\
\hline Precisión & 10 & 10 & 9 & 9 \\
\hline Lenguaje programación & 10 & 10 & 8 & 7 \\
\hline Total & 48 & 48 & 41 & 39 \\
\hline
\end{tabular}

Como variables de entrada del modelo se determinan las notas parciales y la participación de cada estudiante en las actividades académicas y como variables de salida el nivel de riesgo de abandono (alto, medio y bajo). En este modelo la red neuronal realiza una combinatoria de cada posible estado de los atributos de entrada con cada posible estado del atributo a predecir y los arboles de decisión evalúan las condiciones generadas por la red neuronal con el objetivo de clasificar de forma precisa los datos en los rangos que se requieren. Este modelo fue validado utilizando diferentes pruebas matemáticas y actualmente presenta valores accuracy $=0,5338$ y misclass $=0,4662$ (Ver Figura 5) lo que indica que el modelo tiene alta exactitud en sus predicciones.

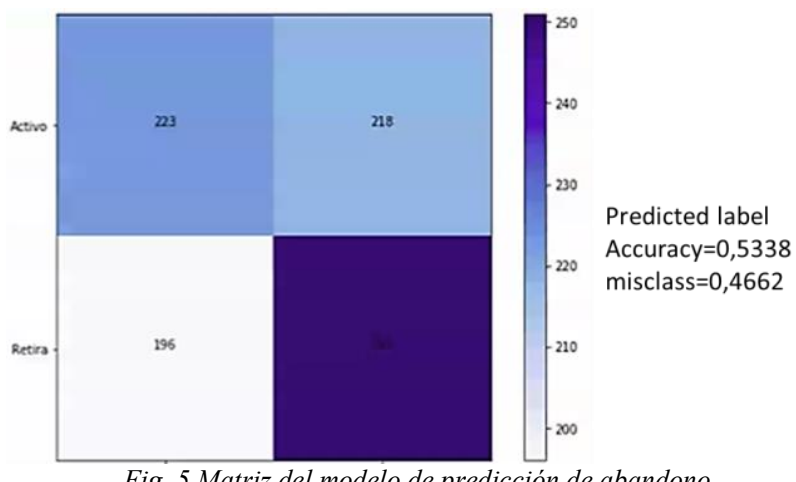

Fig. 5 Matriz del modelo de predicción de abandono 
Este modelo se aplica en cada periodo académico y por cada materia en particular, en la cuarta parte del periodo académico se envía un informe a cada docente en el que se encuentran los estudiantes que tienen un nivel de riesgo alto de abandono, desde ese momento se inicia contacto con estos estudiantes brindándoles actividades de refuerzo y/o acompañamiento según sea el caso. En la mitad del periodo académico el modelo genera las predicciones definitivas. Desde este momento se aplica el programa de contactabilidad con los estudiante que presentan riesgo de abandono, este programa incluye mensajes de apoyo (Ver Figura 6), contacto directo docente-estudiante, actividades de refuerzo, entre otras acciones según sea el caso. Los medios de contacto con los estudiantes pueden ser el correo electrónico, las llamadas o diferentes redes sociales como WhatsApp y Facebook, según lo autorizado por cada estudiante.

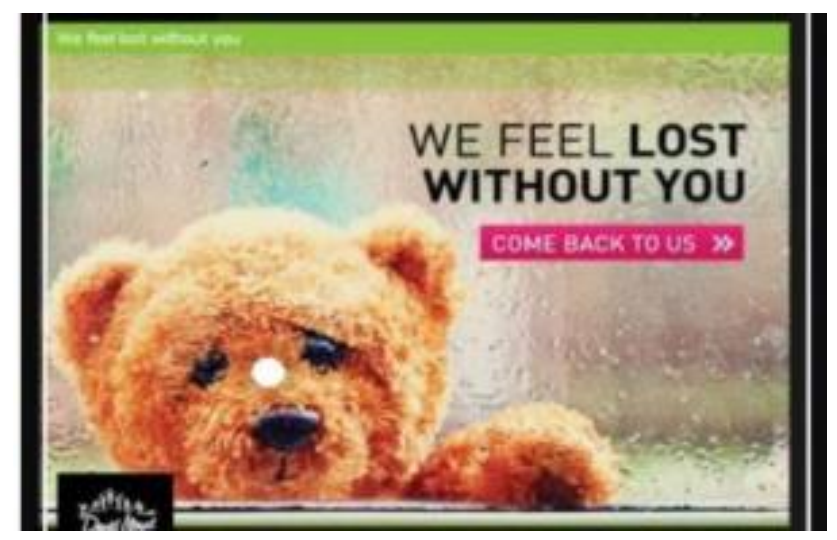

Fig. 6 Ejemplo de mensajes de la plataforma de contactabilidad

\section{Conclusiones}

Las nuevas tecnologías como la gamificación o la inteligencia artificial representan una gran oportunidad al interior de los sistemas de enseñanza, pues al ser implementadas en entornos virtuales de aprendizaje, permiten aumentar la motivación y el engagement, contribuyendo a la disminución del abandono estudiantil y mejorando los procesos de enseñanza y aprendizaje de diferentes temáticas. Por consiguiente, son insumo fundamental para la creación de experiencias de aprendizaje personalizado y eficiente, pues facilitan tener un mayor seguimiento del proceso educativo de cada estudiante, generando un aprendizaje significativo, atractivo e interesante. 


\section{Agradecimientos}

Agradecimiento especial por el apoyo financiero recibido para la realización de este proyecto a la Universidad Industrial de Santander y al Sistema General de Regalías fondos de CTel de la Gobernación de Antioquia administrados a través del Patrimonio Autónomo Fondo Nacional de Financiamiento para la Ciencia, la Tecnología y la Innovación Francisco José de Caldas -MINCIENCIAS-.

\section{Referencias}

Acosta-Medina, J. K., Torres-Barreto, M. L., Alvarez-Melgarejo, M., \& Paba-Medina, M. C. (2019). Desarrollo de competencias ciudadanas en Colombia y en el mundo: contexto actual y estrategias empleadas.〈hal-02305098〉

Alivernini, F., \& Lucidi, F. (2011). Relationship Between Social Context, Self-Efficacy, Motivation, Academic Achievement, and Intention to Drop Out of High School: A Longitudinal Study. The Journal of Educational Research, 104(4), 241-252. https://doi.org/10.1080/00220671003728062

Gonzalez, L., Gomez, M. C., \& Echeverri, J. A. (2017). Motivation and Virtual Education in Computer Science: Case Universidad de Medellín-Colombia. IEEE Latin America Transactions, 15(6), 1176-1181. https://doi.org/10.1109/TLA.2017.7932706

Heublein, U. (2014). Student Drop-out from German Higher Education Institutions. European Journal of Education, 49(4), 497-513. https://doi.org/10.1111/ejed.12097

Kumar, J. M., \& Herger, M. (2013). Gamification at Work: Designing Engaging Business Software. Interaction Design Fundation.

Lassibille, G., \& Navarro Gómez, L. (2008). Why do higher education students drop out? Evidence from Spain. Education Economics, 16(1), 89-105. https://doi.org/10.1080/09645290701523267

Ministerio de Educación Nacional de Colombia. (2017). Reporte sobre deserción y graduación en educación superior. Bogotá, Colombia.

Sastre, S. G., Ortega-Arranz, A., Gómez-Sánchez, E., \& Villagrá-Sobrino, S. (2019). Reflexiones para la introducción de Colaboración y Gamificación en MOOC. Revista Latinoamericana de Tecnología Educativa, 18(1), 163-174. https://doi.org/10.17398/1695-288X.18.1.163

Werbach, K., \& Hunter, D. (2012). For the Win: How Game Thinking Can Revolutionize Your Business. Wharton Digital Press. 